\title{
Current understanding of genetics and genetic testing and information needs and preferences of adults with inherited retinal disease
}

\author{
Martin McKibbin ${ }^{\star, 1}$, Mushtaq Ahmed ${ }^{2}$, Matthew J Allsop ${ }^{3}$, Louise Downey ${ }^{4}$, Richard Gale ${ }^{5}$, \\ Hilary Louise Grant ${ }^{3}$, Barbara Potrata ${ }^{3}$, Thomas A Willis ${ }^{3}$ and Jenny Hewison ${ }^{3}$
}

Advances in sequencing technology and the movement of genetic testing into all areas of medicine will increase opportunities for molecular confirmation of a clinical diagnosis. For health-care professionals without formal genetics training, there is a need to know what patients understand about genetics and genetic testing and their information needs and preferences for the disclosure of genetic testing results. These topics were explored during face-to-face interviews with $\mathbf{5 0}$ adults with inherited retinal disease, selected in order to provide a diversity of opinions. Participants had variable understanding of genetics and genetic testing, including basic concepts such as inheritance patterns and the risk to dependents, and many did not understand the term 'genetic counselling'. Most were keen for extra information on the risk to others, the process for genetic testing and how to share the information with other family members. Participants were divided as to whether genetic testing should be offered at the time of the initial diagnosis or later. Many would prefer the results to be given by face-to-face consultation, supplemented by further information in a format accessible to those with visual impairment. Health-care professionals and either leaflets or websites of trusted agencies were the preferred sources of information. Permission should be sought for disclosure of genetic information to other family members. The information needs of many patients with inherited retinal disease appear to be unmet. An understanding of their information needs and preferences is required to help health-care professionals provide optimal services that meet patient expectations.

European Journal of Human Genetics (2014) 22, 1058-1062; doi:10.1038/ejhg.2013.296; published online 8 January 2014

Keywords: patient understanding; accessible information; inherited retinal disease

\section{INTRODUCTION}

Advances in sequencing technology, particularly the development of next-generation sequencing and the introduction of whole-exome sequencing, are making genetic testing cheaper, faster and more readily accessible. Genetic testing offers a number of potential benefits, even though it may not always identify a treatment or lead to a change in management. Despite these advances and the potential benefits of genetic testing, there is wide variation in access to specialist genetic services and to genetic testing within the publicly funded National Health Service (NHS) in the United Kingdom, particularly for those with inherited retinal disease. ${ }^{1}$ Although the reasons for this are unclear, several factors have been implicated including cost, lack of perceived demand and clinical utility and historic variation in the commissioning and provision of specialist inherited eye disease clinics.

Recent research has begun to demonstrate the demand for and the clinical utility and value of genetic testing for inherited retinal disease. ${ }^{2-5}$ Coupled with planned changes in service delivery so that the commissioning of genetic testing within a specialty, such as ophthalmology, should be led by that specialty, it seems likely that there will be a rapid growth in access to genetic testing for inherited retinal disease. ${ }^{6}$
Patients with inherited retinal disease have variable self-reported understanding of both genetics and genetic testing. ${ }^{7}$ They may also experience more emotional trauma when adjusting to a diagnosis with the likely consequence of progressive visual impairment than to other, more common disorders. ${ }^{8}$ The trauma associated with adjustment can, in part, be reinforced by a lack of information about the cause of the visual impairment. ${ }^{9}$ As a result, health-care professionals need to be aware of the current knowledge and information needs and preferences of adults with inherited retinal disease in order to provide high-quality clinical care. ${ }^{5,10}$

\section{MATERIALS AND METHODS}

Semistructured interviews were conducted with 50 adults with inherited retinal disease to identify the current understanding of genetics and genetic testing, information needs and preferences. Although the focus of the interviews was on diagnostic genetic testing, questions were also asked about predictive testing and about prenatal and pre-implantation genetic diagnosis. Participants were selected from a larger group of 200 study participants, all of whom had completed a prior telephone questionnaire designed to assess their selfreported understanding of and attitudes to genetic testing for inherited retinal disease. Selection for the interviews was based on a purposive sampling frame, constructed to provide a sample with a maximal diversity of self-reported levels

${ }^{1}$ Eye Clinic, Leeds Teaching Hospitals NHS Trust, St James's University Hospital, Leeds, UK; ${ }^{2}$ Yorkshire Regional Genetics, Chapel Allerton Hospital, Leeds, UK; ${ }^{3}$ Leeds Institute of Health Sciences, University of Leeds, Leeds, UK; ${ }^{4}$ Hull and East Yorkshire Eye Hospital, Hull, UK; ${ }^{5}$ Eye Clinic, The York Hospital, York, UK

*Correspondence: M McKibbin, Eye Clinic, Leeds Teaching Hospitals NHS Trust, St James's University Hospital, Leeds LS9 7TF, UK. Tel: +44 113 2066429 ; Fax: +44 113206 6427; E-mail: martin.mckibbin@leedsth.nhs.uk

Received 6 July 2013; revised 7 November 2013; accepted 22 November 2013; published online 8 January 2014 
of knowledge and demographic characteristics. Recruitment to the larger, telephone questionnaire has been described elsewhere. ${ }^{7}$

Face-to-face interviews were conducted with the 50 participants using a semistructured interview guide. Interviews were recorded, transcribed verbatim, analysed and coded by two researchers using a thematic approach. ${ }^{11,12}$ To ensure consistency, two researchers analysed a number of randomly selected transcripts for the emerging themes independently, coded the data, and compared codes and themes. Any disagreements were resolved by consensus. The researchers continued with the analysis of the remaining interviews and met at regular intervals to agree or refine themes. Analysis was undertaken using a constant comparison and contrastive approach. Looking for negative cases further refined understanding and relationships between and within the themes.

Participants gave written, informed consent before the interviews. The project was approved by the Leeds (East) Research Ethics Committee (10/H1306/90).

\section{RESULTS}

Sample

The baseline characteristics of the 50 participants are given in Table 1 . Participants with a range of clinical diagnoses, including both congenital and acquired visual impairment, and inheritance patterns were included. The most common clinical diagnoses were retinitis pigmentosa in 22 participants and macular dystrophy in 20 participants, of whom seven had Stargardt disease and four had Best disease.

Understanding of genetics, genetic testing and genetic counselling In line with the sampling strategy, understanding of genetics was variable and several participants felt that they had only a limited understanding. (P111: 'My understanding of it is quite vague. I go to my brother because he's quite bright! He knows more scientific stuff than me. I've done a little (research) and I understand a little but not in great depth'). Some participants felt that their understanding of their personal diagnosis or inheritance pattern was good. (P86: 'I've got a faulty gene that means that my eye doesn't get rid of waste

\section{Table 1 Baseline characteristics of the $\mathbf{5 0}$ study participants}

\begin{tabular}{|c|c|}
\hline Mean age & 45.7 years (SD 16) \\
\hline \multicolumn{2}{|l|}{ Sex } \\
\hline Male & 25 \\
\hline Female & 25 \\
\hline \multicolumn{2}{|l|}{ Ethnicity } \\
\hline White British & 39 \\
\hline British Asian or other & 11 \\
\hline \multicolumn{2}{|l|}{ Highest level of education } \\
\hline To GCSE or O level & 13 \\
\hline To college or beyond & 37 \\
\hline \multicolumn{2}{|l|}{ Sight impairment certification status } \\
\hline Sight impaired or severely sight impaired & 40 \\
\hline Not certified or unknown & 10 \\
\hline \multicolumn{2}{|l|}{ Parenting status } \\
\hline Have or plan to have children & 38 \\
\hline No plans to have children & 12 \\
\hline \multicolumn{2}{|l|}{ Other affected family members } \\
\hline Yes & 29 \\
\hline No & 21 \\
\hline
\end{tabular}

products and it causes new blood vessels to grow and...leak and haemorrhage and they will cause a scar and they will wipe out the central area. So the mutation on the gene that I have is the one that causes the eye condition'). Others perceived their understanding to be poor. Inheritance patterns were often confused or misunderstood. (P7: 'Dominant is the one that's the...most important and... recessive.... is the one that isn't so common and important' and P197: 'Dominant is when somebody has got a stronger...gene... that's more rich and has more of an impact than the recessive one').

Some participants believed that certain inherited conditions were the result of consanguineous marriage but did not seem to understand the genetic basis. (P197: 'He didn't say it was genetic but he said it's because you married in the family'). Understanding of the risk to other family members was also variable. Some felt that a condition could only be genetic if all the family members were affected. Many participants confused the risk to future generations, failing to understand that the risk applied to each pregnancy, for example, or believing that the risk to a child with two carrier parents was one in two. (P64: 'My basic understanding of recessive is you need two carriers and if two carriers of the gene match up, then there is a 50-50 chance that...those genes will combine and will pass that condition on').

Although several participants were unclear about what a genetic test involved, many understood that genetic testing could offer a range of potential benefits, including: confirmation or provision of a diagnosis and the pattern of inheritance, help to plan the future, assisting clinical research and identifying future treatments. (P11: 'It's for providing the individual with information .... and... because the more information they have about the population and different variants, the more they can do their testing on possible remedies and cures'). For those participants without other known affected family members, genetic testing was valued as giving confirmation that the condition was indeed genetic.

Participants were also concerned about possible negative aspects of genetic testing, including emotional consequences, implications for other family members and the possible impact on employment and insurance. (P211: 'Whenever there's a genetic disease... you know it's been passed down from your parents somewhere. So...there's a level of guilt with parents and there's a level of blame from the children's side'). Some wondered if genetic testing was relevant to them, particularly if it failed to add any new information or to identify a new treatment or cure. (P201: 'It's nice to know... a little bit more about my eye condition but it doesn't really move me any further on and there's no treatment available for what I have').

Several participants were aware that the role of a genetic counsellor included the provision of support and information, especially in relation to genetic testing and the results. (P172: 'I think they help you to understand the risk... of... you being a carrier and passing on the gene to any family that you might decide to have' and P185: 'They explain...the likelihood of inheriting a particular disease from your parents... and then help you through the impact of that'). However, many were confused about the role, believing it to be more limited to that of providing support and psychological counselling and feeling therefore that it was not relevant to them. (P121: 'Somebody...I can phone... having that extra bit of support would be really lovely' and P125: 'I don't do all this counselling stuff because it's just speaking to somebody who knows nothing').

\section{Information needs and preferred sources of information}

Nearly all participants wanted to have a precise diagnosis of their eye condition and to know the consequences. Of particular importance 
was a desire to know and understand the pattern of inheritance and the risk to their children. (P24: 'The most important thing is ... how is it going to affect me... is it going to affect my family...'). Some participants were keen to understand the role of genetic testing in relation to carrier status testing and highlighted the need for the information to be provided sensitively. (P197: 'If you are contemplating marrying a family member...it's not targeting any religion or something').

In relation to genetic testing, participants often wanted more information about what a genetic test involved, what would happen to the sample and how testing could be provided. (P115: 'You need to know the implications of the test... what it is that they're actually going to test for, how long it will take ... and then obviously the result... how that's going to be delivered ... who's going to give it to me'). Before accessing genetic testing, participants wanted to have additional information not only on the process of genetic testing and the possible positive and negative aspects, but also on the accuracy or reliability of testing. (P130: 'If you are going to have an information sheet... it should tell... how accurate any information that might be given could be or how reliable').

To obtain information about genetics and genetic testing, healthcare professionals were the preferred source for most participants. Ideally this would be done at the time of clinic review. (P207: 'Faceto-face would be better because I can understand more when somebody's talking to me rather than reading it from a bit of paper... They can explain what it actually means'). However, a recurring theme was the need to provide this information using simple, easily understood language and then to provide it supplementary information either in a leaflet format at the time of consultation or by letter after the consultation. (P100: 'Just an explanation... making it easy for the less academic of us to understand ... doctors use too many long words... you're no further forward sometimes...s so put it in plain English' and P184: 'The explanatio$\mathrm{n}$... was very interesting but I've forgotten most of it because it was given verbally... so to have it in some kind of leaflet format...that people could refer back to, would be very useful'). Some also noted that the provision of information before a genetic eye clinic appointment would be helpful. This was felt to provide an opportunity to consider the issues beforehand so that questions could be brought along to the clinic consultation. Other sources of information included the internet, particularly the websites of national charities, and social networking sites. Although some participants found online information to be the most helpful, others expressed a concern about the accuracy, detail and conflicting information available through an internet search. (P45: 'A lot more online... more than the hospita1... Support groups on Facebook...that's probably my biggest source of information' and P95: 'There are too many things (and) the information is often too in-depth and not lay friendly'). The role of health-care professionals in identifying 'trusted' websites was acknowledged.

Most participants wanted printed information, but several commented that their visual impairment often made it difficult to access. (P110: 'A lot of the information I get from the NHS is in writing. I keep telling them there is no point in sending me a letter. The font size is just size 12. I can't see it!'). Although some participants had additional hearing impairment, others preferred to receive information in an audio format. Several expressed enthusiasm for the use of interactive CDs or websites, highlighting additional benefits over fixed print or audio formats. (P110: 'Very often you deal with this on your own and you don't have anybody to bounce your thoughts off...Something more interactive...I would have found useful... especially if you are typing an answer and the real answer comes up and you think... actually I forgot that bit... I didn't know that bit'). Positive case histories and the use of 'frequently asked questions' were also suggested as being helpful, but were often unavailable.

\section{Timing of genetic testing and disclosure of information}

Although most participants supported the provision of genetic testing for inherited retinal disease, some questioned whether it would offer them any particular benefit. Most often this was because their family was already complete, the diagnosis was not in doubt or because visual impairment was already severe. (P187: 'I'm not really that interested at this point in my life...I've had my children... it's a done deal...it's a fait accompli'). Participants were divided as to the ideal time for genetic testing to be offered. Some felt it should be offered at the time of the initial diagnosis of the cause of visual impairment. This view was more common in those with other affected family members. Others felt that genetic testing should not be offered until after the initial diagnosis, possibly not until an individual was in a stable relationship or planning to start a family. (P25: 'Maybe a bit later... so you can digest what you've been told first...then offer genetic testing...maybe at the next appointment or a couple of appointments down the line').

Some participants felt that the results of genetic testing could be given to them by letter, as they may only confirm what had been discussed at a prior clinic consultation. Most, however, were keen that the results should be given to them during a clinic review or via internet telephony so that there would be the opportunity to ask questions and to get help understanding the implications. (P111: 'I don't think it should be done over the phone because... I think if you need support... you can't always ask questions that you want to ask over the phone. So... I do think it's better to be face to face'). Provided the results were given by a health-care professional who understood the result and the implications, participants did not seem to express a preference as to which member of the health-care team this should be. Many wanted the results and any supplementary information in a letter to help them retain the relevant information.

Most participants were aware that a genetic diagnosis would have implications not only for them but also for other family members, both with and without relevant symptoms. On receiving a positive genetic test result, some felt that they would feel obliged to share the information with other family members, but were aware that they may feel anxious about testing and wondered how family members would deal with a positive predictive test result and whether they would want to undergo testing themselves. Many reported that they did not know how to approach the subject with relatives, particularly children, and would welcome guidance. (P201: 'How do I talk about it with my son... keep that sort of open dialogue ... no one's given me any guidance... how would you discuss this with your children?'). Several thought that their relatives should be given accurate information by a doctor. (P55: 'I think the doctor should explain what it is that's wrong, explain the hereditary nature... how it manifests itself and everything else'). Many felt that health-care professionals should be able to share genetic information relating to one family member with other affected people in the same family, but most would prefer to be asked for their consent for such information to be shared.

\section{DISCUSSION}

This study provides data on the current understanding of genetics and the information needs and preferences of adults with inherited retinal 
disease. Although most participants were recruited from eye clinics or were actively engaged with national charities, their understanding was variable and many were keen to have additional information, particularly in relation to the risk to other family members and the process for genetic testing. Health-care professionals and national charities were the preferred sources of information.

Interviewees were selected from a larger group of 200 participants in order to reflect a diversity of backgrounds and opinions. The findings of this study suggest that, individuals with inherited retinal disease do not differ significantly from the general population in terms of their understanding of genetics and genetic testing and their information needs. ${ }^{13,14}$ Telephone questionnaires with the larger group of participants identified that a self-reported understanding was greater in those with higher educational achievement, suggesting that the need for information is greater in those with lower educational achievement or that they are less confident. ${ }^{7}$ Access to information may also be more difficult for those with lower educational achievement. The findings suggest that either or both of the initial provision or the recall of information may have been poor. In order to meet patient needs and expectations, it would seem sensible to match the detail of information provided to the individual, using plain and sensitive language and identifying and addressing any misconceptions. ${ }^{15,16}$

In particular, participants wanted an accurate diagnosis of their eye disease and also information on the risk to other family members, practical aspects of genetic testing and advice on how to share information with others. However, they often did not seem to understand the role of a genetic counsellor, suggesting that healthcare professionals may need to be proactive in recommending genetic counselling.

Although several felt that genetic information and testing should be available at the time of diagnosis, some did not feel that such information was of relevance to them. Information on the risk to other family members appeared to be valued most by those whose families were not yet complete. A few participants felt that they would prefer not to know about the risk to children until they were in a stable relationship. Klitzmann and Sweeney ${ }^{17}$ identified similar concerns over how and when to disclose genetic risk information. Most participants could suggest a number of potential benefits to genetic testing, but were aware that it may not change the management of their eye disease. This suggests that the personal utility of genetic testing for inherited retinal disease is greater than the clinical utility.

Participants wanted accessible information that would supplement verbal information provided at the time of clinic review. The best practice for communicating with the visually impaired suggests an acknowledgement of print, email or audio preferences for appointments and communication and the use of black pens, accessible computer screens or tactile diagrams to aid understanding. ${ }^{18}$ These ideas could be used for supplementary information. In many countries, there is a legal obligation to provide accessible information to those with visual impairment and this may also help tackle social isolation and depression. Patients with inherited retinal disease often want ongoing support and review, with additional information about daily living aids, employment, mobility and research updates. $5,10,16$

Most participants felt that the best sources of information were either health-care professionals or leaflets and websites of national charities. Although the internet is a powerful resource for information provision, people can find it confusing and overwhelming. Ophthalmic information can be an amalgamation of differing views, may feature commercial bias and be poorly accessible to those with visual impairment. ${ }^{19-21}$ Patients may want and need directing to reliable sources of information. ${ }^{5,20}$ Current preferences appear to be either the websites of national charities or sites hosted by professional bodies. ${ }^{22}$ Such organisations may be best placed to provide accurate information, reviewed by professionals and that is transparent, accessible and updated regularly. Social networking sites are increasingly accessible and popular with visually impaired people, but are not always felt to be an appropriate resource for obtaining answers to personal questions. ${ }^{23}$ Possibly given the mean age of the sample, smartphone applications were not mentioned as a useful source of information.

Most participants would prefer to receive the results of genetic testing during a face-to-face consultation, followed by a letter. Opinions on intra-familial disclosure were much more divided: many felt that they would prefer to inform other members of a positive genetic test result, knowing the individual personalities and family dynamics better, but others did not feel that other members of the family should be informed. Attitudes to disclosure appear to be influenced by a desire to protect the individual and family members, the perceived relevance or usefulness of the information and the nature of the relationships within the family. ${ }^{24,25}$ The desire for assistance with intra-familial communication and the role of health-care professionals in providing support, identifying other sources of information and acting as a focal point for referral have already been identified. ${ }^{14,24,25}$ Although both patients and the public seem keen to know the results of genetic testing that may be relevant to them, this enthusiasm is reduced for non-fatal and untreatable disease. As a minimum therefore, it would seem sensible for professionals to ask permission from those with inherited retinal disease before the results of genetic testing are shared with other family members. ${ }^{26}$

The findings of this project are limited by the small sample size, the fact that most of the participants were actively engaged with healthcare professionals or national charities and the focus on patients within the United Kingdom. Most of the participants had not had prior access to diagnostic genetic testing as a result of local commissioning policy, but many would have been approached to provide a sample of DNA for research analysis. The sample also included participants with a variety of clinical diagnoses and with differing durations of visual impairment. However, participants were chosen from a larger cohort and the purposive sampling frame ensured that those selected for the interviews reflected not only a diversity of self-reported levels of understanding but also a varied demographic background. For this reason, the findings may be broadly applicable to others with a range of inherited retinal diseases, both those who regularly attend the clinic review and those who are not actively engaged with eye care services.

Many patients with inherited retinal disease currently have a poor understanding of genetics, genetic testing and genetic counselling. Most are keen to have more information, particularly about patterns of inheritance, the risk to children, practical aspects of genetic testing and communication of the risk to other family members. Health-care professionals and national charities are the preferred sources of information. Verbal information should be supplemented by additional material, accessible to those with visual impairment. At present, the information needs of those with inherited retinal disease appear to be unmet.

\section{CONFLICT OF INTEREST}

The authors declare no conflict of interest. 


\section{ACKNOWLEDGEMENTS}

This paper presents independent research funded by the National Institute for Health Research (NIHR) under its Research for Patient Benefit (RfPB) Programme (Grant Reference Number PB-PG-0909-20228). The views expressed are those of the author(s) and not necessarily those of the NHS, the NIHR or the Department of Health.

1 Moore AT, Burton H: Genetic ophthalmology in focus: a needs assessment and review of specialist services for genetic eye disorders. PHG Foundation, 2008. Available at http://www.phgfoundation.org/reports/4984/. (Last accessed 21 May 2013)

2 Combs R, McAllister M, Payne $\mathrm{K}$ et al: Understanding the impact of genetic testing for inherited retinal dystrophy. Eur J Hum Genet 2013; 21: 1209-1213.

3 Downs K, Zacks DN, Caruso R et al: Molecular testing for hereditary retinal disease as part of clinical care. Arch Ophthalmol 2007; 125: 252-258.

4 Pradhan M, Hayes I, Vincent A: An audit of genetic testing in diagnosis of inherited retinal disorders: a prerequisite for gene-specific intervention. Clin Experiment Ophthalmol 2009; 37: 703-711.

5 Mezer E, Babul-Hirji R, Wise R et al: Attitudes regarding predictive testing for retinitis pigmentosa. Ophthalmic Genet 2007; 28: 9-15.

6 Human Genome Strategy Group (HGSG). Building on our Inheritance: Genomic Technology in Healthcare 2012. Available at https:/www.gov.uk/government/ uploads/system/uploads/attachment_data/file/134568/dh_132382.pdf. (Last accessed May 2013)

7 Willis TA, Potrata B, Ahmed M et al: Understanding of and attitudes to genetic testing for inherited retinal disease-a patient perspective. Br J Ophthalmol 2013; 97 $1148-1154$.

8 Jangra D, Ganesh A, Thackray R et al: Psychosocial adjustment to visual loss in patients with retinitis pigmentosa. Ophthalmic Genet 2007; 28: 25-30.

9 Branham K, Yashar B: Providing comprehensive genetic-based ophthalmic care. Clin Genet 2013; 84: 183-189.

10 Sutherland JE, Day MA: Genetic counseling and genetic testing in ophthalmology. Curr Opin Ophthalmol 2009; 20: 343-350.

11 Daly J, Kellehear A, Gliksman M: The Public Health Researcher: A Methodological Approach. Melbourne, Australia: Oxford University Press, 1997; pp 611-618.
12 Braun V, Clarke V: Using thematic analysis in psychology. Qual Res Psychol 2006; 3 77-101.

13 Lanie AD, Jayaratne TE, Sheldon JP et al: Exploring the public understanding of basic genetic concepts. J Genet Couns 2004; 13: 305-320.

14 Condit CM: Public understandings of genetics and health. Clin Genet 2010; 77: 1-9.

15 Biotechnology Australia. Genetics in Family Medicine: The Australian Handbook for General Practitioners 2007. Available at http://www.nhmrc.gov.au/files nhmrc/file/ your_health/egenetics/practioners/gems/sections/17_genetics_in\%20practice.pdf. (Last accessed June 2013)

16 Combs R, Hall G, Payne $\mathrm{K}$ et al: Understanding the expectations of patients with inherited retinal dystrophies. Br J Ophthalmol 2013; 97: 1057-1061.

17 Klitzman RL, Sweeney MM: 'In sickness and in health'? Disclosures of genetic risks in dating. J Genet Couns 2011; 20: 98-112.

18 Hall G, Clarke A: Communicating with clients who are visually impaired; In: Wiggins J, Middleton A (eds). Getting the Message Across: Communicating with the Diverse Population in Clinical Genetics. Oxford: Oxford Press, 2013; pp 26-41.

19 Schalnus R, Aulmann G, Hellenbrecht A, Hägele M, Ohrloff C, Lüchtenberg M: Content quality of ophthalmic information on the internet. Ophthalmologica 2010; 224: 30-37.

20 Kahana A, Gottlieb JL: Ophthalmology on the internet: what do our patients find? Arch Ophthalmol 2004; 122: 380-382.

21 Lüchtenberg M, Kuhli-Hattenbach C, Sinangin Y, Ohrloff C, Schalnus R: Accessibility of health information on the internet to the visually impaired user. Ophthalmologica 2008; 222: 187-193.

22 Smallcombe KA, Kearns LS, Mackey DA: Introducing a new retinitis pigmentosa patient information website. Clin Experiment Ophthalmol 2005; 33: 226-228.

23 Brady E, Zhong Y, Morris MR, Bigham JP: Investigating the appropriateness of socia network question asking as a resource for blind users. Proceedings of the 2013 conference on Computer Supported Cooperative Work 2013. Available at http://research.microsoft.com/pubs/180051/cscw2013_vizwiz_social.pdf. (Last accessed June 2013).

24 Wiens ME, Wilson BJ, Honeywell C, Etchegary $\mathrm{H}$ : A family genetic risk communication framework: guiding tool development in genetics health services. J Community Genet 2013; 4: 233-242.

25 Gallo AM, Angst DB, Knafl KA: Disclosure of genetic information within families. Am J Nurs 2009; 109: 65-69.

26 Wolff K, Brun W, Kvale G, Ehrencrona H, Soller M, Nordin K: How to handle genetic information: a comparison of attitudes among patients and the general population. Public Health Genomics 2010; 13: 396-405. 